\title{
Complexity based on synchrosqueezing analysis in gear diagnosis
}

\author{
Yacine Imaouchen $^{1}$, Rezak Almama $^{1}$ and Marc Thomas ${ }^{2, a}$ \\ 1 Electrical Engineering Laboratory, Faculty of Technology, Université de Bejaia, 06000 Bejaia, Algeria \\ 2 Department of Mechanical Engineering, École de Technologie Supérieure. 1100, Notre-Dame Street West, Montreal, \\ H3C 1K3 Quebec, Canada
}

Received 5 March 2015, Accepted 14 April 2015

\begin{abstract}
The Synchrosqueezing is a special case of the reassignment method and concentrates the timefrequency representation (TFR) in a scale dimension. Compared to other TFR enhancement methods, the synchrosqueezing offers better adaptability, less deformation for IF profile and an exact reconstruction formula for constituent components. This paper deals with the investigation of descriptors based on the combination of the synchrosqueezing transform (SST) and Lempel-Ziv complexity methods. This last one transforms the analyzed signal into a data sequence. In the first part, the vibration signal components are extracted by using the synchrosqueezing transform and the reconstruction method. Afterward, the LempelZiv complexity values are calculated. Since the complexity values are not dependent on the magnitude of the measured signal, the proposed method is less sensitive to the data sets measured under different data acquisition conditions. This approach is applied for monitoring and diagnosing the defects during a fatigue test on a first gear reducer and also when varying the load on a second gear reducer by using the recorded vibration signals. It can also provide a new way for feature extraction and recognition of gear system faults.
\end{abstract}

Key words: Lempel-Ziv complexity / synchrosqueezing / fault diagnosis / vibration / gear defect

\section{Introduction}

Gear vibration signals are commonly modelled as a sum of AM/FM components. The meshing frequency and its harmonic components are modulated by the shaft rotational frequency and its harmonics for a faulty gearbox. This is often identified via detecting the presence of the sidebands [1]. As the understanding of the theory advanced, amplitude and frequency-modulation (AM-FM) decompositions have been applied in a large range of problems: they are usually analyzed by the short-time Fourier transform (STFT) [2] or the continuous Wavelet transform (CWT) [3], while most quadratic methods can be seen as variations of the Wigner-Ville distribution (WVD), with squared STFTs (spectrograms) and WTs (scalograms) as special cases. It is well-known that both transforms for such signals draw strips in the timefrequency $(\mathrm{TF})$ or time-scale (TS) plane, centered at the ridges corresponding to the instantaneous frequencies [4]. Kodera et al. [5] proposed the Modified Moving Window Method in the STFT. Their approach takes into account the phase information to enhance the dispersion of the STFT magnitude. In addition, the Wigner Distributions can be adapted to have a perfect localization of the signal

\footnotetext{
${ }^{a}$ Corresponding author: marc.thomas@etsmtl.ca
}

with specific frequency modulations. However, some limitations in using these techniques were sometimes encountered. As an example, resolution problems in both time and frequency depending on the frequency range when trying to locate sharp peaks and low frequency features. To avoid the difficulties encountered by implementing these two methods, Auger and Flandrin [6,7] presented an efficient method for computing the times and frequencies for the reassigned spectrogram without explicitly computing the partial derivatives of phases. Hence, the reassignment is substituted by a combination of STFTs with appropriate windows. The Synchrosqueezing transform, introduced in references [8-10], is a special case of the reassignment method that purposes to refine a timescale representation, while remaining invertible. Its purpose is similar to that of reassignment with the further improvement of allowing for reconstruction. Relatively to mechanical fault diagnosing, the feature of two sideband frequencies close to the fundamental frequency of rotor fault signal or the feature of the AM-FM gearbox fault signal cannot be directly extracted from the blurred time-frequency representation (TFR). This is caused by the occurrence of noise and transients from faulty machine components. This can be addressed by considering the extraction of meaningful components from the mixed signals. The other way is to keep the signals as they evolve without decom- 
posing them into different components and instead make use of the TF analysis provided by the SST method. To analyse the TF features of the stator current signal, $\mathrm{Pu}$ et al. [11] performed the SST method in order to detect and diagnose the rotor faults. Hazra et al. [12] applied the SST for monitoring and diagnosing the gearbox faults, where Chuan et al. [13] brought another alternative to provide the TFR by implementing the generalised SST to detect gear faults. All of them studied the instantaneous frequency profile with concentrated TFR offered by the SST method. As announced in Daubechies et al. [8], the SST method can be used as a method to decompose a vibration signal like the EMD method $[14,15]$; we propose to benefit from this advantage to diagnose gear faults. This paper is composed in two sections. The first part gives a thorough discussion of the TFRs, and their properties, providing all the information that is needed to understand and apply them effectively then the procedure to use the SST method like EMD to decompose a multicomponent signal are exposed. In addition, the complexity approach is more detailed and made in a comprehensive procedure to calculate it. The second part deals with the performance of the proposed method on the experimental gear vibration signals.

\section{Signal processing}

\subsection{Synchrosqueezing based Wavelet transform}

A multicomponent signal can be considered as expressed by:

$$
x(t)=\sum_{m=1}^{M} x_{m}(t)+r(t), \quad \text { with } \quad x_{m}(t)=a_{m}(t) \mathrm{e}^{i \phi_{m}(t)}
$$

for some finite $M$, where $a_{m}(t)>0$ is a continuously differentiable function, $\phi_{m}(t)$ is a two times continuously differentiable function satisfying $\phi_{m}^{\prime}(t)>0$ and $\phi_{m+1}^{\prime}(t)>\phi_{m}^{\prime}(t)$ for all $t . r(t)$ is a residual signal, which represents noise or measurement error.

Synchrosqueezing as presented by Daubechies et al. [8], is a nonlinear operator that sharpens the timefrequency plot of a signal's continuous wavelet transform and enhances the TFR in a manner similar to reassignment method [9], but it still enables mode retrieval as in the empirical mode decomposition (EMD) $[14,15]$. So the CWT of the analytic signal $x(t)$ is given by:

$$
W_{x}(a, b)=\frac{1}{\sqrt{a}} \int_{-\infty}^{\infty} x\left(t \overline{\psi\left(\frac{t-b}{a}\right)} \mathrm{d} t\right.
$$

where $a$ is the scale, $b$ the time offset, and $\psi$ a properly chosen analytical mother wavelet that is concentrated on the positive frequency axis.

Then, the instantaneous frequency $\omega_{x}(a, b)$ assuming $W_{x}(a, b) \neq 0$ can be derived as:

$$
\omega_{x}(a, b)=\frac{-j}{W_{x}(a, b)} \frac{\partial W_{x}(a, b)}{\partial b}
$$

During the frequency mapping, $(a, b) \rightarrow\left(\omega_{x}(a, b)\right.$, $b)[8,16]$, the synchrosqueezing is applied to reassign the time-scale representation to the $\mathrm{TF}$ plane as:

$$
T_{x}\left(\omega_{l}, b\right)=\frac{1}{\Delta \omega} \sum_{a_{k}:\left|\omega\left(a_{k}, b\right)-\omega_{l}\right| \leqslant \frac{\Delta \omega}{2}} W_{x}\left(a_{k}, b\right) a_{k}^{-3 / 2}(\Delta a)_{k}
$$

where $\omega_{l}$ is the $l$ th discrete angular frequency, $a_{k}$ is the $k$ th discrete scale point, $\Delta \omega=\omega_{l}-\omega_{l-1}$ and $(\Delta a)_{k}=$ $a_{k}-a_{k-1}$.

Finally, the instantaneous angular frequency is normalized by $2 \pi$ as IF $f=\frac{\omega}{2 \pi}$. To reconstruct the signal, two ways are principally used, direct and ridges reconstruction $[16,17]$. It is important to know that ridge reconstruction appears to be more robust to interference and noise, while direct reconstruction performs better in the case of considerable amplitude/frequency modulation [18]. This is quite understandable, since, as we saw, the AM/FM components can be represented as a sum of tones with particular amplitude, phase and frequency relationships, so the amplitude and frequency modulation can be viewed as arising due to interference between these AM/FM induced tones.

\subsection{SST performance}

\subsubsection{Simulated signal (principle of gearbox diagnosis)}

When a defect occurs in a gearbox, the meshing frequency (and its harmonics) will be modulated by the fault shaft's rotation frequency or its harmonics. The vibration signal of the faulty gearbox can thus be expressed as [19]:

$$
\begin{aligned}
x(t)= & \sum_{m=1}^{M} x_{m}(t) \\
= & \sum_{m=1}^{M} A_{m}\left(1+B_{m} \cos \left(2 \pi m f_{s} t\right)\right) \\
& \times \cos \left(2 \pi m Z f_{s} t+\phi(t)\right)+r(t)
\end{aligned}
$$

where $M$ is the number of the vibration harmonics, $A_{m}$ is the amplitude of the $m$ th meshing harmonic, $B_{m}$ is the modulus of the $m$ th amplitude modulation, $f_{s}$ is the rotational frequency, $Z$ is the number of teeth, and $\phi(t)$ is the frequency modulation function. $r(t)$ is a residual signal that presents the noise or measurement error.

A simulated gearbox signal is analyzed as follows to test the performance of the proposed method. According to Equation (5), the simulated signal of the fault gear has been chosen similar with three harmonics:

$$
x(t)=x_{1}(t)+x_{2}(t)+x_{3}(t)+\text { noise }
$$

where $A_{1}=1.8, A_{2}=1.6, A_{3}=1.3, B_{1}=2.8, B_{2}=$ $2.4, B_{3}=2.2, Z=20$ and $f_{s}=25 \mathrm{~Hz}$, so the meshing frequency for $x_{1}(t)$ is $500 \mathrm{~Hz}$. A white noise is added to the signal with the $\mathrm{SNR}=1.52 \mathrm{~dB}$. 
(a)
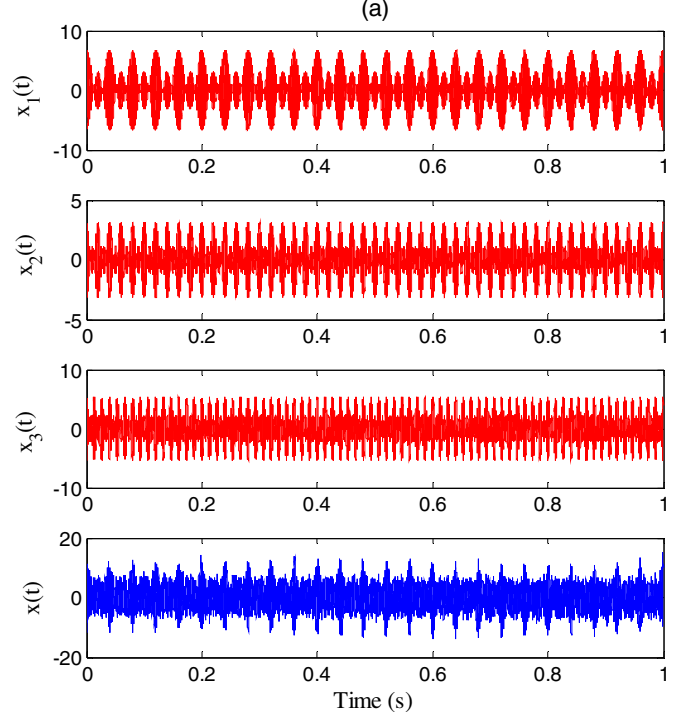
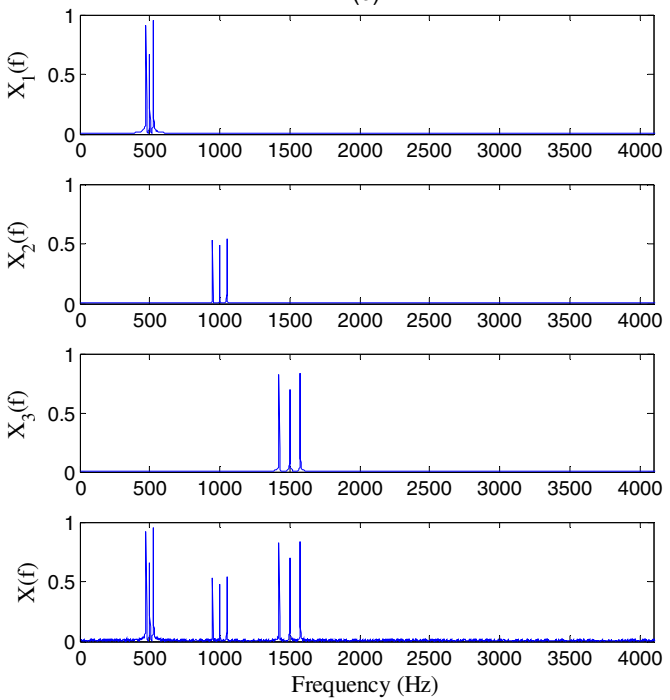

Fig. 1. (a) The three components and the synthetic signal and (b) their respective spectrums.

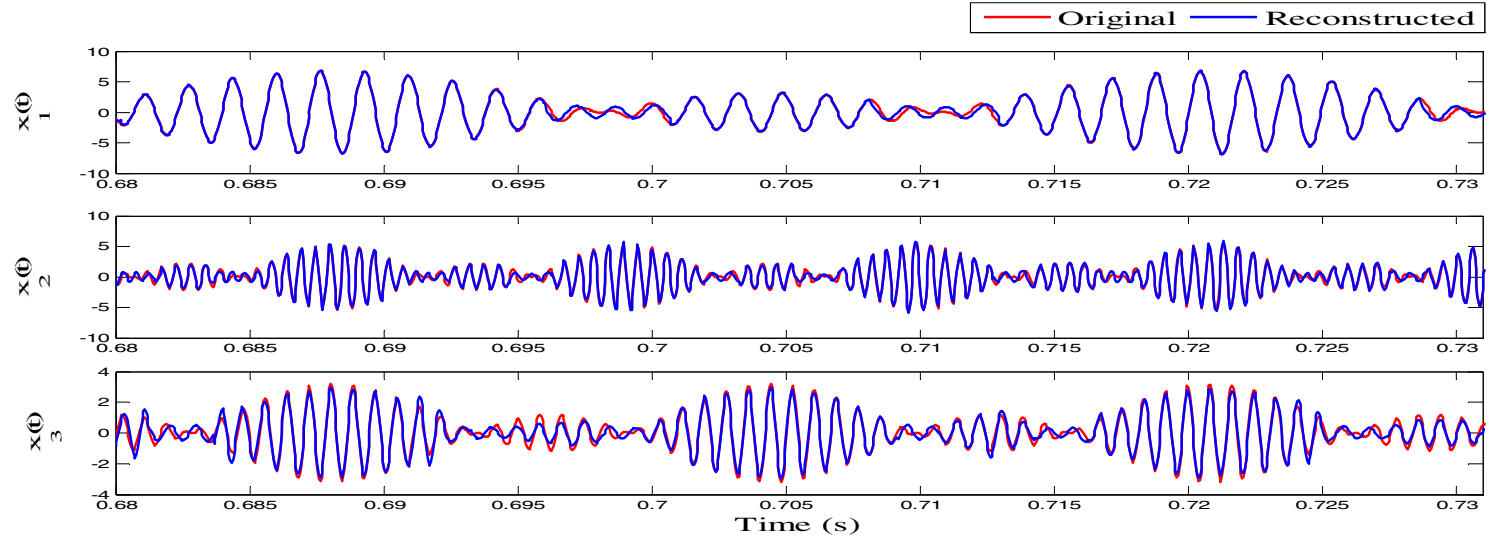

Fig. 2. The original components of $x(t)$ (red color) and the three components obtained by the SST method (blue color).

The raw signal $x(t)$ is sampled at $4096 \mathrm{~Hz}$ for $1 \mathrm{~s}$. The signal composed by the three components and the noise are plotted in Figure 1 with their respective spectrums. The three original components are compared to those retrieved by the SST method (see Fig. 2). The zoom is applied on features to see the effectiveness of the method to reconstruct exactly the same components.

\subsubsection{Real gear vibration signal}

To improve the efficiency of the SST method to extract the exact components from real signals, a gear vibration signal obtained during a fatigue test performed by CETIM (Senlis, Fr) [15, 20-22] is decomposed by the method. Figure 3 shows the real signal $x(t)$, its first four retrieved modes $(R M \mathrm{~s})$ and their respective spectrums. As we can see, the frequency spectrum of each component $R M_{i}$ is also found on the signal spectrum. For instance, the first component $R M_{1}$ reports the first harmonic of the meshing frequency $f_{\mathrm{e}}=340 \mathrm{~Hz}$ with the sideband frequencies.

\subsection{Complexity analysis}

The complexity analysis is based on the Lempel-Ziv (LZ) definition $[14,23,24]$. Before calculating the LZ complexity measure $C(n)$, the signal must be transformed into a finite symbol sequence. This approach transforms the analysed signal into a data sequence. In the context of a gear vibration signal with a known mean value, typically the discrete-time signal $x(n)$ is converted into a new binary sequence $S(n)$. This new sequence is rebuilt by comparing the value of each sample of the previous sequence $x(n)$ within the mean value. If the value of the sample is larger, it is set to one, otherwise to zero. In order to compute LZ complexity, the sequence $S(n)$ is scanned from left to right and the complexity counter is increased by one unit every time a new subsequence of consecutive characters is encountered. Thus, the LempelZiv complexity reflects the number of all different subsequences contained in the original sequence. Figure 4 (reproduced from [24]) explains the procedure of calculating the Lempel-Ziv complexity for a finite sequence of length $N$, where $C_{U L, N}=N / \log _{2}(N)$. For generality 
(a)
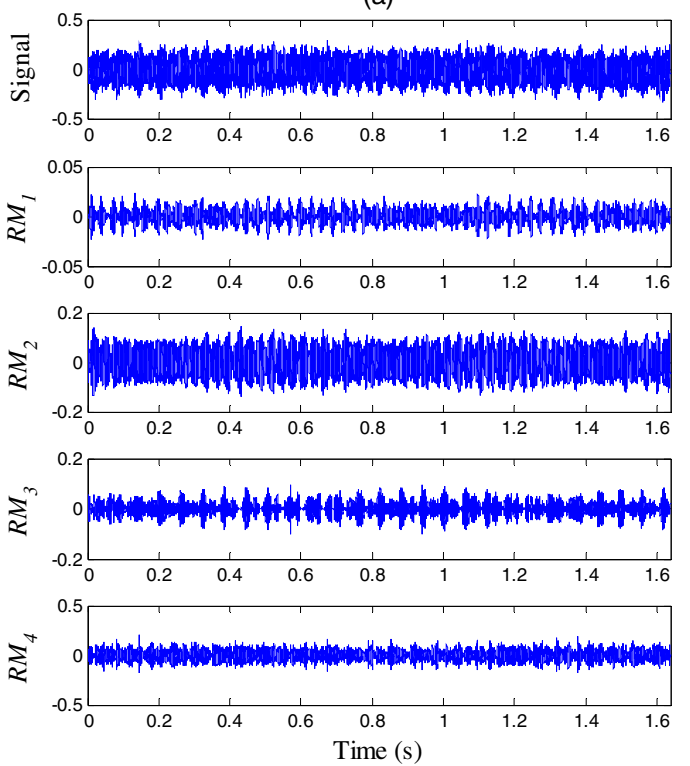

(b)
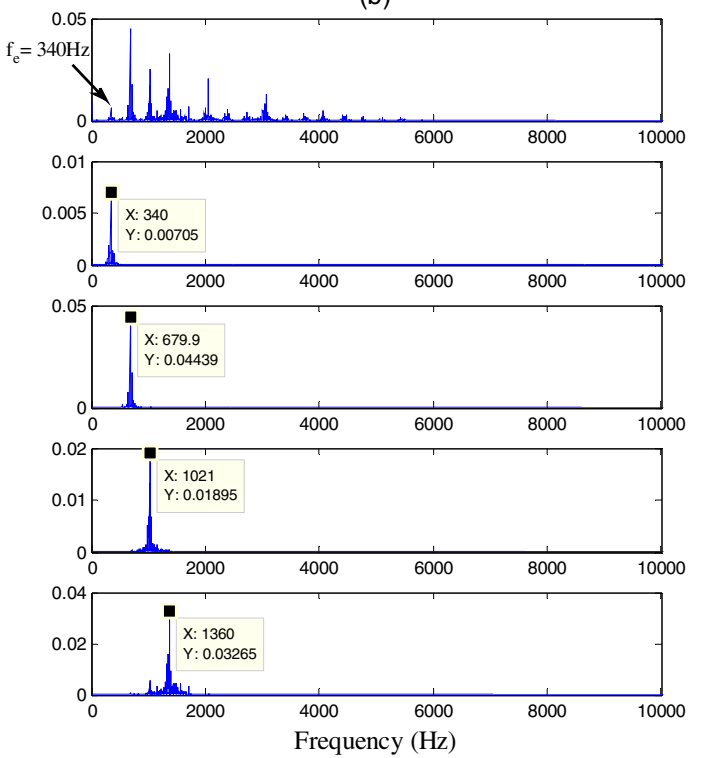

Fig. 3. (a) Real gear vibration signal $x(t)$ with its first four $R M \mathrm{~s}$, and (b) their respective spectrums.

sake, normalized complexity $C(n)$ is often used to obtain a measure independent of the sequence length. As an example, we consider a real gearbox vibration signal constituted by 10000 samples and the rate sampling is $20 \mathrm{kHz}$. The sampled signal in Figure 5a represents the sequence $x(n)$. To obtain a new binary sequence $S(n)$, each sample of $x(n)$ is compared to the mean of $x(n)$. If the value of the sample is larger, it is set to one, otherwise to zero. The new sequence $S(n)$ is shown in Figure $5 \mathrm{~b}$. The LZ complexity reflects the number of all different subsequences contained in this new sequence. Following the steps explained above in the diagram (Fig. 4), 296 subsequences were obtained and the normalized complexity value for this example can be calculated by:

$$
C(n)=\frac{c(n)}{N / \log _{2}(N)}=\frac{296}{10000 / \log _{2}(10000)}=0.3933
$$

As mentioned in references [23, 24] the $c(n)$ calculation can be viewed as independent of the data sequence length if the data length considered is greater than $N$ points. To confirm this assumption, two real gear vibration signals are studied to examine the effect of the number of data samples. The rate sampling of the first signal is $20 \mathrm{kHz}[15,20-22]$, and $24 \mathrm{kHz}$ for the second [25]. The normalised LZ complexity of both signals was computed following the procedure shown in Figure 4. As revealed in Figure 6 , the normalized complexity remains invariable since the data length $N>6000$.

\section{Experimental tests}

In this part we propose an approach based on the SST method and the Normalized LZ Complexity. Two different bases of gear vibration signals are used to corroborate this focus.

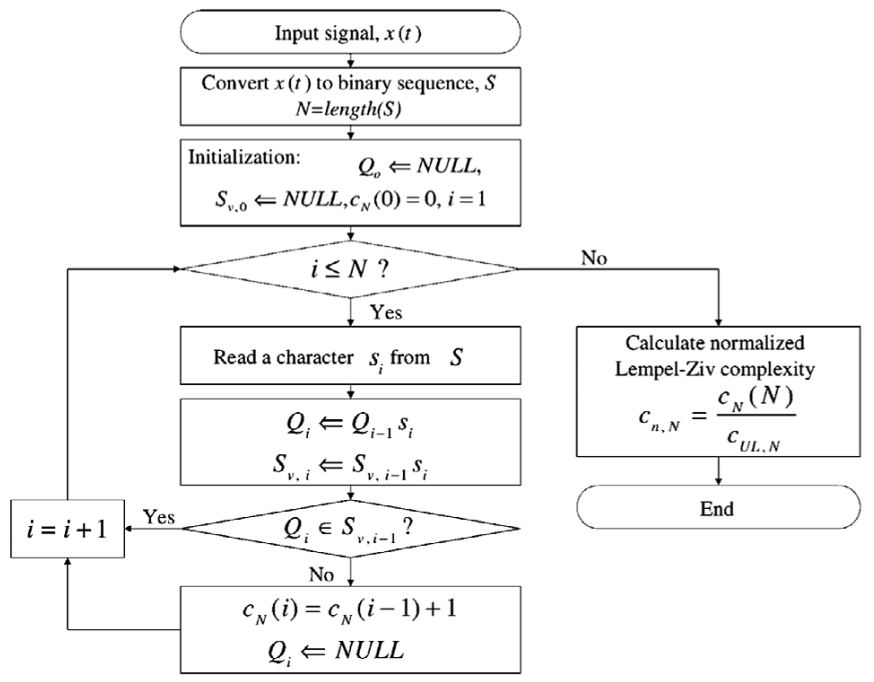

Fig. 4. Block diagram for calculating the Lempel-Ziv complexity.

\subsection{Application 1: fatigue test}

SST and complexity methods were performed for monitoring and diagnosing the defects during a fatigue test performed by CETIM (Senlis, Fr) on a gear reducer by using the recorded vibration signals $[15,20-22]$. The number of teeth is respectively 20 teeth on the first wheel and 21 teeth on the second wheel. The rotation speed of the input shaft of the reducer is $1000 \mathrm{rpm}$ which gives rotation frequencies of $f_{1}=16.67 \mathrm{~Hz}$ and $f_{2}=15.87 \mathrm{~Hz}$ on the output shaft. The meshing frequency is $f_{e}=333.33 \mathrm{~Hz}$. The experiment was carried out for a period of 12 days. During experimentation, the testing gear was drived from a well operating state to a deteriorated gear state. Every 
(a)

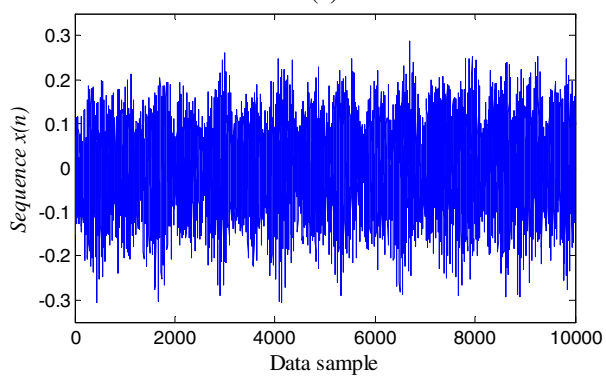

(b)

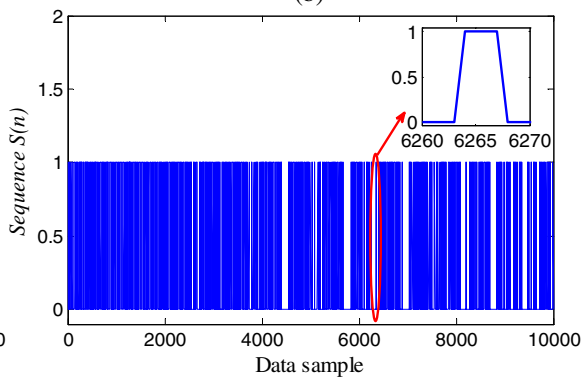

Fig. 5. Gear vibration signal: (a) sequence $x(n)$, and (b) the new data sequence $S(n)$.

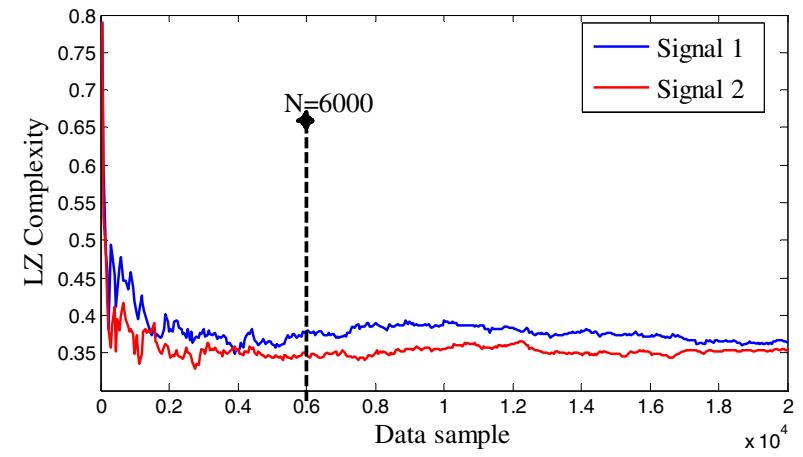

Fig. 6. The effect of data length on the normalized complexity.

Table 1. Daily mechanical appraisal (from CETIM, FR).

\begin{tabular}{cc}
\hline Day & Observation \\
\hline 0 & No acquisition \\
1 & No anomaly \\
2 & No anomaly \\
3 & $/ / / /$ \\
4 & $/ / / /$ \\
5 & $/ / / /$ \\
6 & Chipping teeth $1 / 2$ \\
7 & No evolution \\
8 & Tooth $1 / 2:$ no evolution \\
& Tooth $15 / 16:$ start chipping \\
9 & Evolution of the chipping of the teeth $15 / 16$ \\
1 & $/ / / / /$ \\
11 & Chipping across the full width of the tooth \\
12 &
\end{tabular}

day, the test bench is stopped after recording the vibration signals in order to correlate the gear teeth state with the measured signals (Tab. 1).

\section{$\mathrm{G}$}

ear $(1 / 2)$ chipping was observed at the 6 th day and gear $(15 / 16)$ spalling was observed since the 8th day. On the last day, the damage was in an advanced stage close to the breakage of two teeth. The data recording was set-up with the following parameters: the length of records was 60000 samples and the sampling frequency is $20 \mathrm{kHz}$. In this study, the proposed method is performed on 32768 samples. The meshing signal is principally caused by shocks between teeth of wheels that compose the reducer. The meshing signal is modulated in amplitude and frequency by the signals emitted from the two wheels whose frequencies are respectively $f_{1}$ and $f_{2}$.

\subsubsection{Statistical study}

Traditionnaly, time statistical descriptors used in industry are RMS, Peak to Peak, Crest Factor and Kurtosis [26]. In order to investigate their performance, RMS and kurtosis of vibration signals were computed in order to follow gear fault development for the 12 days of operation. It may be observed in Figure 7 that Kurtosis becomes sensitive only the 10th day of operation and that RMS is not very sensitive until the 11 th day.

\subsubsection{Proposed method}

To enhance the damage detection, this part proposes to combine two methods: the SST and the normalized LZ complexity. The procedure follows these steps:

1. Perform the SST method to obtain the retrieved modes $(R M)$ for the 12 days signals.

2. Obtain the energy and Kurtosis in each $R M$ and find the $\alpha$ best modes for each day signal by maximizing energy and kurtosis.

3. Compute normalized complexity of each day signal $C(x(n))$ and each $R M, C\left(R M_{i}(n)\right)$.

4. Calculate the kurtosis for each day signal $K u(x(n))$ and each extracted mode $K u\left(R M_{i}(n)\right)$.

5. Finally, Equation (8) gives the complexity-Kurtosis ratio (indicator) for each day signal $x(n)$.

indicator $=\left(\frac{\sum_{i=1}^{\alpha} C_{i}\left(R M_{i}(n)\right)}{C(x(n))}\right) /\left(\frac{\sum_{i=1}^{\alpha} K u_{i}\left(R M_{i}(n)\right)}{K u(x(n))}\right)$

To select $\alpha$ best modes (step 2), the energy level is used to obtain the RM with maximum energy [24]. The energy contained in all RMs is calculated by:

$$
E_{x(n)}=\sum_{i=1}^{N} E_{R M_{i}(n)}
$$



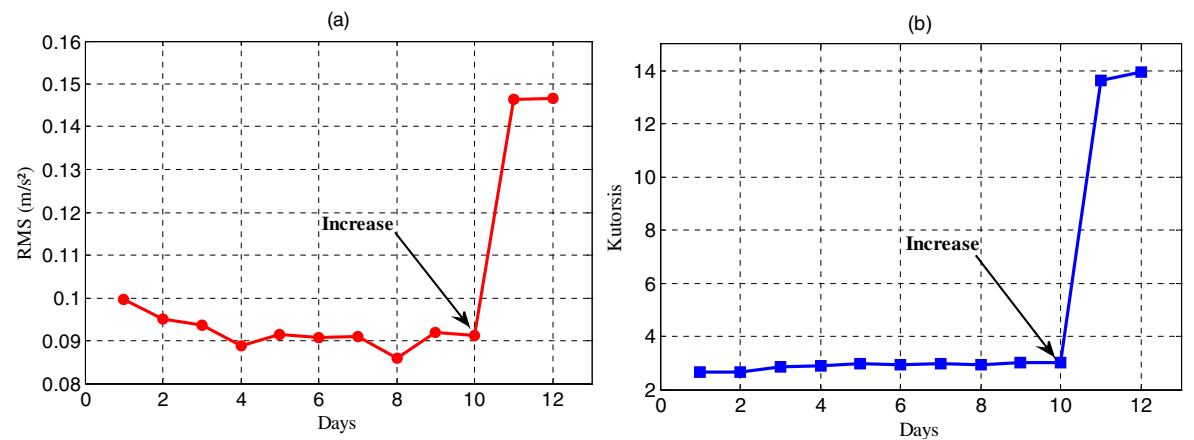

Fig. 7. Time indicators: Kurtosis and RMS values of the raw signals.

where $N$ represents the number of all RMs, and $E_{R M_{i}(n)}$ represents the amount of energy contained in the $i$ th RM which is defined as:

$$
E_{R M_{i}(n)}=\sum_{t=1}^{\alpha}\left|R M_{i}(n)\right|^{2}
$$

The $R M \mathrm{~s}$ that contain relatively the highest amount of energy are selected as the representative RMs since faultrelated RMs contain a higher amount of energy than the rest of the RMs [27]. However, the only use of energy level is not a good criterion to select the interest RM because a higher energy level is also related with higher retrieved modes of the SST. As known mechanical faults like gear faults produce impulses in the measured faulty signals. The energy level does not always reflect this nature while it is calculated based on the amplitude of the RM instead of the impulsiveness of a signal [24]. To overcome this shortcoming in this study, the energy and the kurtosis criteria are jointly used for modes selection since the kurtosis is a good indicator of impulsiveness. Thus, the best RM that reflects the signal nature of the high energy and the impulsiveness should be selected. The best RMs can be found by:

$$
R M_{\text {best }}=\left\{R M \mid \frac{d E_{R M}}{d R M}=0 \cap \max \left(K_{R M}\right) \cap K_{R M}>3\right\} .
$$

The complexity-Kurtosis ratio is applied for the 12 days signals. Figure 8 shows the evolution of this descriptor established for the vibration signals to detect the damage evolution. If we accept a normal variation of the indicator between 0.36 and 0.43 with a mean at 0.39 , the results show that the descriptor level starts to decrease with the default evolution since the 8th day and a more sensitive degradation after due to a greater damage at the 11 th and the 12th day. When a defect appears and becomes stronger, more impacts would be generated when the defected tooth touches the others, leading to a longer period of defect-related vibration. More frequency components will be contained within the frequency spectrum then the complexity and kurtosis values change. As a result, the Complexity-Kurtosis ratio will decrease.

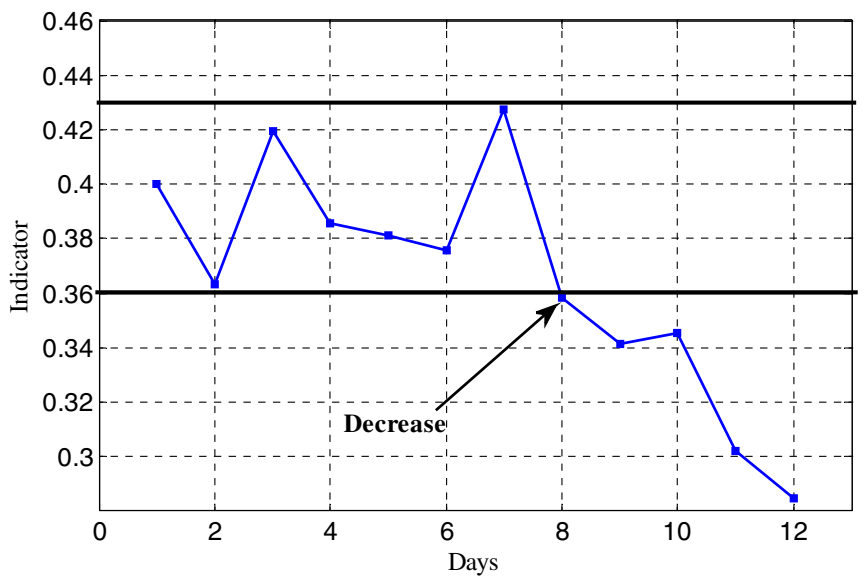

Fig. 8. Proposed indicator values of the SST retrieved components.

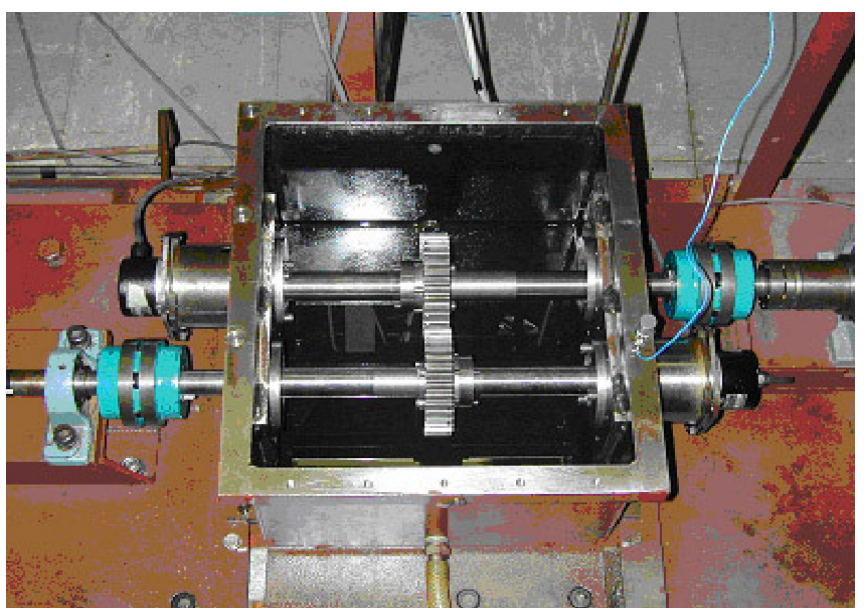

Fig. 9. Experimental test of Peter Rig [22].

\subsection{Application 2: dependence on load}

To confirm the assessment, the proposed approach is applied to gear vibration signals of the experimental test of Peter Rig (University of New Wouth Wales, Kensington, Australia and LASPI Senlis, Fr [25]), see Figure 9. We are interested on the first campaign of tests where the number of teeth was $32: 32$ with a defected tooth on 

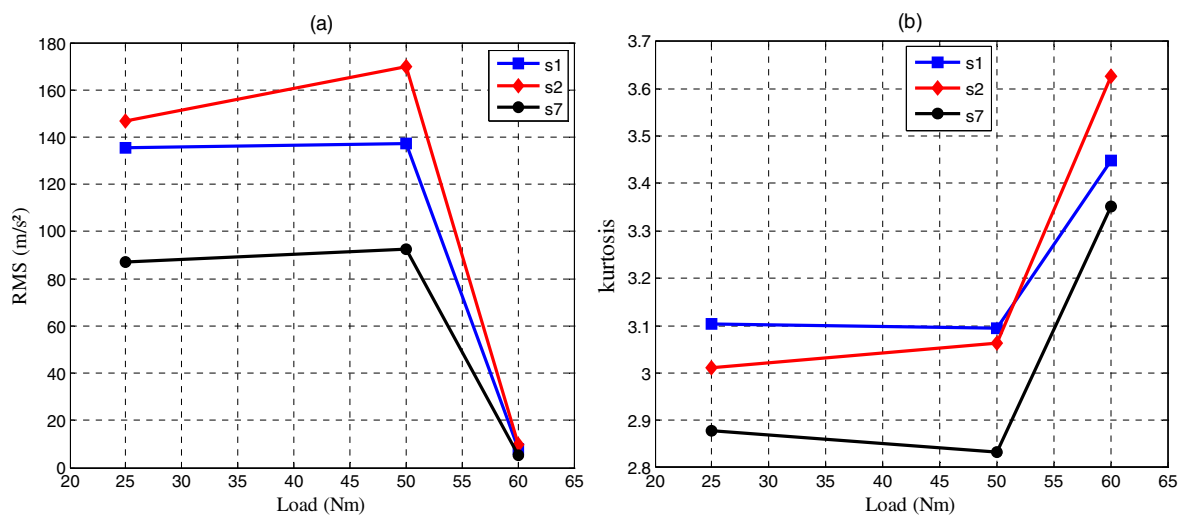

Fig. 10. Time indicators: (a) RMS and (b) kurtosis of the raw signals (speed: $600 \mathrm{rpm}$ ).

the driven wheel. Different sensors were used to record the data signals on the gear reducer; 7 accelerometers and 1 tachymeter. The data length for vibration signals is 100000 samples and $24 \mathrm{kHz}$ as the sampling frequency. In this study the measurement of three sensors (s1, s2 and $\mathrm{s} 7$ ) is considered with three load values; 25,50 and $60 \mathrm{Nm}$ and $10 \mathrm{~Hz}$ as the rotating frequency. Thus, three measurements are recorded for each sensor to evaluate the effect of load on the gear fault signals and the data length used to compute the normalized complexity is 32768 samples.

\subsubsection{Statistical study}

The time indicators: RMS and Kurtosis are computed for each case. Figure 10 illustrates the evolution of the two descriptors. The RMS descriptor rises gradually and falls under different load values at $600 \mathrm{rpm}$ rotational speed. Contrary to the RMS, the Kurtosis drops then rises considerably with the increase of the load values. Since, the evolutions of these descriptors are not enough sensitive under loads changes.

\subsubsection{The proposed method}

Following the five steps of the proposed method below (Sect. 3.1.2), the same procedure is applied. For each sensor three measures was recorded;

1. Perform the SST method for each record to obtain the $R M \mathrm{~s}$.

2. Maximize the energy and the Kurtosis for each $R M$ and find the $\alpha$ best modes.

3. Compute normalized complexity of record signal $C(x(n))$ and each $R M, C\left(R M_{i}(n)\right)$.

4. Calculate the Kurtosis for each record signal $K u(x(n))$ and each retrieved mode $K u\left(R M_{i}(n)\right)$.

5. Finally, compute the Indicator values like Equation (8) do.

Three features are obtained in Figure 11 respectively for each sensor (1, 2 and 7 ). The proposed indicator reveals an amplitude decrease matching the load values

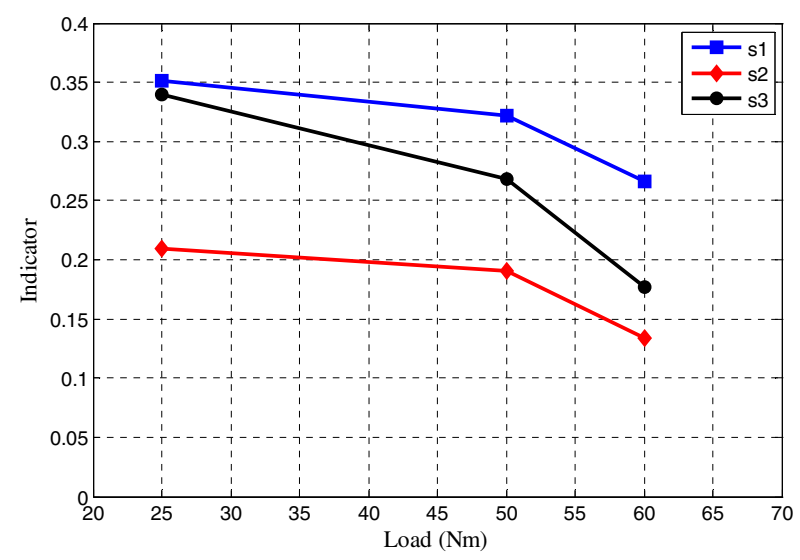

Fig. 11. The effect of load on the proposed indicator (speed: $600 \mathrm{rpm})$.

increase. The indicator seems also more sensitive to the load changes. When the load increases, the impact of the defected tooth touching the others teeth gain in amplitude, influencing the frequency components of the signal and more sideband frequencies will be added to the other frequency components. So, the normalized complexity of the recorded signal changes in value. In the other hand, the impulsiveness will be more intensive and the kurtosis value increases. Finally, the indicator ratio swings and reacts with falling gradually.

\section{Conclusion}

This study presents an investigation of the Synchrosqueezing method and the Normalized Complexity for monitoring the gear fault evolution. First, numerical signals were studied to perform the effectiveness of the SST method to decompose a gear simulated signal. The robustness of the method towards noise was established by the perfect correlation between the retrieved and the original components. Secondly, this method is applied to vibration data obtained from two experimental tests; fatigue test conducted by CETIM (France) during 12 days and Peter Rig conducted by the UNWK (Australia). The 
Lempel-Ziv complexity, a non-dimensional index ranging roughly between 0 and 1 , is jointly considered with the Kurtosis (indicator of impulsiveness). A new descriptor based on the complexity-Kurtosis ratio was developed and performed on the retrieved modes signals obtained by the SST method and adopted as a fault severity measurement. Regarding the test fatigue, standards methods such as RMS and Kurtosis were sensitive to the defect only from the 10th day, while the proposed descriptor appears to be sensitive since the beginning of the spalling from the 8th day. In the other hand, when applied on vibration signals recorded from the Peter rig, this descriptor is revealed to be sensitive to the variation of load. The results indicate that the fault severity can be assessed reasonably well by the proposed method.

\section{References}

[1] W.D. Mark, Stationary transducer response to planetarygear vibration excitation II: effects of torque modulations, Mech. Syst. Signal Process. 23 (2009) 2253-2259

[2] B. Badri, M. Thomas, S. Sassi, Variable Drive Frequency Effect on Spindle Dynamic Behavior in High Speed Machining, CMMNO, 547-554

[3] I. Daubechies, The wavelet transform, time-frequency localization and signal analysis, IEEE Trans. Inform. Theory 36 (1990) 961-1005

[4] I. Yesilyurt, The application of the conditional moments analysis to gearbox fault detection - a comparative study using the spectrogram and scalogram, NDT \& E International 37 (2004) 309-320

[5] K. Kodera, C.D. Villedary, R. Gendrin, A new method for the numerical analysis of nonstationary signals, Phys. Earth and Plan. Int. 12 (1976) 142-150

[6] F. Auger, P. Flandrin, Improving the Readability of Time-Frequency and Time-Scale Representations by Reassignment Methods, IEEE Trans. Signal Proc. 43 (1995) 1068-1089

[7] F. Auger, E. Chassande-Mottin, P. Flandrin, Making reassignment adjustable: The Levenberg-Marquardt approach, in Proc. IEEE-ICASSP, Kyoto, 2012, pp. 38893892

[8] I. Daubechies, J. Lu, H.T. Wu, Synchrosqueezed wavelet transforms: an empirical mode decomposition-like tool, Appl. Comput. Harmonic Anal. 30 (2011) 243-261

[9] F. Auger, P. Flandrin, Y.T. Lin, S. McLaughlin, S. Meignen, T. Oberlin, H.T. Wu, Time-Frequency Reassignment and Synchrosqueezing: a review, IEEE Signal Process. Magazine 30 (2012) 32-41

[10] G. Thakur, E. Brevdo, N.S. Fuckar, H.T. Wu, The Synchrosqueezing algorithm for time-varying spectral analysis: Robustness properties and new paleoclimate applications, Signal Process. 93 (2013) 1079-1094

[11] T. Pu, L. Chai, Y.X. Sheng, Z.-X. Liu, A Synchrosqueezing Transform Based Method for Rotor Fault Diagnosis of Squirrel-cage Induction Motors, IEEE Conference Publication, Proceedings of the 32nd Chinese Control Conference, Xi'an, China, Jully 26-28, 2013

[12] B. Hazra, S. Narasimhan, Rotating machinery diagnosis using synchro-squeezing transform based feature analysis. Proceedings of MFPT Conference, Cleveland, Ohio, USA, May 13-17, 2013
[13] L. Chuan, L. Ming, Time-frequency signal analysis for gearbox fault diagnosis using a generalized synchrosqueezing transform, Mech. Syst. Signal Process. 26 (2012) 205-217

[14] M. Kedadouche, T. Kidar, M. Thomas, A. Tahan, M. El Badaoui, R. Guilbault, Combining EMD and Lempel-Ziv Complexity for early detection of gear cracks, International Conference Surveillance 7, Chartres, France, Oct 29-30, 2013

[15] T. Kidar, M. Thomas, R. Guilbault, M. Elbadaoui, Comparison between the efficiency of LMD and EMD algorithms for early detection of gear defects, Mechanics \& Industry 14 (2013) 121-127

[16] G. Thakur, H.T. Wu, Synchrosqueezing-based recovery of instantaneous frequency from non-uniform samples, SIAM J. Math. Analys. 43 (2011) 2078-2095

[17] S. Meignen, T. Oberlin, S. Mclaughlin, A new algorithm for multicomponent signal analysis based on synchrosqueezing: with an application to signal sampling and denoising, IEEE Trans. Signal Process. 60 (2012) 57875798

[18] D. Iatsenko, P.V.E. McClintock, A. Stefanovska, Linear and synchrosqueezed time-frequency representations revisited. Part I: Overview, standards of use, related issues and algorithms. Digital Signal Processing (2014), [preprint: arXiv: 1310.7215]

[19] F.Q. Peng, D.J. Yu, J.S. Luo, Sparse signal decomposition method based on multiscale chirplet and its application to the fault diagnosis of gearboxes, Mech. Syst. Signal Process. 25 (2011) 549-557

[20] M. El Badaoui, Contribution of vibratory diagnostic of gearbox by Cepstral analysis, (in french). Ph.D. thesis, Jean Monnet University of St Etienne (FR) (1999)

[21] A. Pareya, M. El Badaoui, F. Guillet, N. Tandon, Dynamic modelling of spur gear pair and application of empirical mode decomposition-based statistical analysis for early detection of localized tooth defect, J. Sound Vib. 294 (2006) 547-561

[22] M. El Badaoui, F. Guillet, J. Daniere, New applications of the real cepstrum to gear signals, including definition of a robust fault indicator, Mech. Syst. Signal Process. 18 (2004) 1031-1046

[23] R. Yan, R.X. Gao, Complexity as a Measure for Machine Health Evaluation, IEEE Trans. Instrumentation Measurement 53 (2004) 1327-1334

[24] H. Hong, M. Liang, Fault severity assessment for rolling element bearings using the Lempel-Ziv complexity and continuous wavelet transform, J. Sound Vib. 320 (2009) $452-468$

[25] N. Sawalhi, R.B. Randall, Simulating gear and bearing interactions in the presence of faults Part II: Simulation of the vibrations produced by extended bearing faults, Mech. Syst. Signal Process. 22 (2008) 1952-1966

[26] S. Sassi, B. Badri, M. Thomas, Tracking surface degradation of ball bearings by means of new time domain scalar descriptors, Int. J. COMADEM 11 (2008) 36-45

[27] R. Yan, R.X. Gao, Rotary machine health diagnosis based on empirical mode decomposition, J. Vib. Acousti. 130 (2008) 021007 